\title{
Review on Cloud Computing: Issues, Services and Models
}

\author{
Neeti Thakur \\ TIE IT, Bhopal-India
}

\author{
Dhananjay Bisen \\ UIT, RGPV, Bhopal, India
}

\author{
Vikas Rohit \\ UIT, RGPV, Bhopal, India
}

\author{
Neelesh Gupta \\ T I E IT, Bhopal-India
}

\begin{abstract}
Cloud computing is Internet-based technology. It provides ondemand resources, software and information sharing by internet to users and devices. Cloud computing is the product of traditional network technology like grid computing, distributed computing and parallel computing. It is growing every day due to its basic services such as virtual pool of resources and its services, which are provided to the users through Internet. This paper is providing the basic description of cloud models and service of cloud computing. Paper also represents the cloud computing issues and its basic comparison with mobile and grid computing.
\end{abstract}

\section{Keywords}

Cloud computing, security, privacy, issue.

\section{INTRODUCTION}

Long ago a single computer was used in data center and its data was shared by multiple users and it controlled activities of all shared users but that technology increases hardware complexity of architecture. This constraint resulted in evolution of new technology CLOUD COMPUTING. Cloud computing [2] is an advanced or forward looking system architecture which derives the future of computing. This system provides advanced features of operating system, client servers and browsers. This technology provides multiple resources to users like storage, services, networks and applications with reducing the complexity of system. This technology works successfully without any need of physical connection between resources. This technology is also known as on demand computing or use and pay computing. The "cloud" in cloud computing could be characterized as the set of systems, space, administrations, and interfaces that join together to convey parts of computing as an administration. Cloud administrations incorporate the conveyance of programming, foundation, and space over the Internet dependent upon client request.

In cloud computing [3] data and programs stored in cloud and can be accessed by clients and light weighted mobile devices anywhere anytime. This carries many advantages including data pervasiveness, affability of access and flexibility. Cloud computing will be used in future as a new mode of business computing. The main concept of cloud computing is to bring down the processing burden on users by improving the storage and data handling capability of the cloud. It allows user terminal to provide input and output devices, and powerful capability of computing of cloud according to the demand. All of this only can be happened through a simple internet connection using a standard browser or other connection. Cloud computing uses virtualization technology with self-service ability for computing via internet.

In another way it also increases security. Cloud computing provides much better up to data security technologies as compare to the data owner can. However, since cloud computing providers may be able to afford much batter secure data outside of the control of the data owner but it inevitably acquaints security issues too. A recent survey shows that data security and privacy risks have become the primary concern for people to shift to cloud computing. Cloud computing security [4] deals with all facts of making cloud computing secure. Most of the facts are not unique for cloud setting: data in cloud shows the possibility of being attacked. Therefore, cloud computing security embrace all aspects of computing security, including the design of security architectures, minimization of attack, protection from malware, and enforcement of access control. As Where this technology reduces complexity of large systems on the other hand it has some risks and disadvantages also like security, insecure interface and sharing of resources and attacks.

\section{PAPER ORGANIZATION}

This paper presents review of cloud computing on the basis of their issues, services and models. The rest of the paper is organized as follows: In Section III, literature survey of cloud computing have discussed. In Section IV, it presents a basic deployment models such as public, private, community, hybrid. Section V, VI, VII, VIII, XI deals with service models, issues, comparison with grid and mobile computing and final conclusion respectively.

\section{LITERATURE SURVEY}

Some works have been presented in recent years on the Profitability of using cloud computing services in order to improve the performance of running large scientific applications. Cloud based services indeed claim that they can achieve significant cost savings over owned computational resources, due to the pay-per-use approach and reduced costs in maintenance and administration which are spread over a large user basis [3]. Until recently, most of the scientific tasks were run on clusters and grids, and many works explored how to optimize the performance of scientific applications in such specific contexts. However, the cloud is not a completely new concept with respect to grids, it indeed has intricate connections to the grid computing paradigm and other technologies such as utility and cluster computing, as well as with distributed systems in general. Several works investigated several different aspects involved in running scientific workflows in the cloud, for instance focusing on optimal data placement inside the cloud, the overall experience and main issues faced when the cloud is used and the suitability of cloud storage systems such as Amazon S3 for the scientific community.

In 2013 Giuseppe Aceto, Alessio Botta, Walter de Donato, Antonio Pescap presented Survey Paper on Cloud monitoring [2]. Survey starts with analyzing motivation for cloud monitoring and analyzes the properties of a monitoring system for the cloud. Finally, identify open issues, main challenges and future directions in the field of cloud monitoring. It have also discussed the main activities in cloud environment that have strong benefit from or actual need of monitoring. 
In 2011 Guannan HU and Wenhao ZHU introduced a dynamic user-integrated cloud computing architecture [6]. This architecture 2 integrates clients with storage capacity and computing competency to data center dynamically, it expands the scale of cloud computing data center. Collaboration of clients with the data center provides services to the other users.

Bhaskar Prasad Rimal, Eunmi Choi and Lan Lumb presented A taxonomy and survey of Cloud Computing System in 2009 [11]. This paper shows a comprehensive taxonomy for describing cloud computing architecture and applies this survey to existing cloud computing services developed by various projects such as Google, Amazon. This paper also identified areas requiring further research.

Panagiotis Kalagiakos, Panagiotis Karampelas illustrated research paper on Cloud Computing Learning [9]. This paper describe cloud computing ecosystem as a new opportunity in designing cloud computing educational platforms where learning actors can reuse learning resources handled by cloud educational operating systems. To enhance learning objects portability and interoperability not only cloud computing API standards should be advocated by the key cloud providers but also learning resources standards should be defined by the Open Cloud Computing Education Federation as proposed by this paper.

\section{DEPLOYMENT MODELS}

Cloud computing [5] is the collection of virtualized and scalable resources and capable of hosting application which is also providing on demand scalability and guaranteed quality of services. Where this technology reduces complexity of large systems on the other hand it has some risks and disadvantages also like security, insecure interface and sharing of resources and attacks. On demand self service, broad network access, resource pooling, automatically control and optimize resources are the uniqueness of cloud computing.

\subsection{Private Cloud}

The cloud infrastructure is owned or possessed by an organization and operated for that one and only organization. Private cloud computing system provides virtualization solution and target on centralized IT services. Private cloud hold full control over collective data, security and system performance private cloud is a small scale cloud as compare to public cloud [10].

\subsection{Community Cloud}

The cloud infrastructure is shared by several organizations and all shared organizations have similar missions, objectives, security requirement and policy. Community cloud contains software, data storage and computing resources shared by multiple other organizations. The infrastructure is used entirely by a group of organization which makes community cloud different from public cloud. It provides greater cost savings than the private cloud.

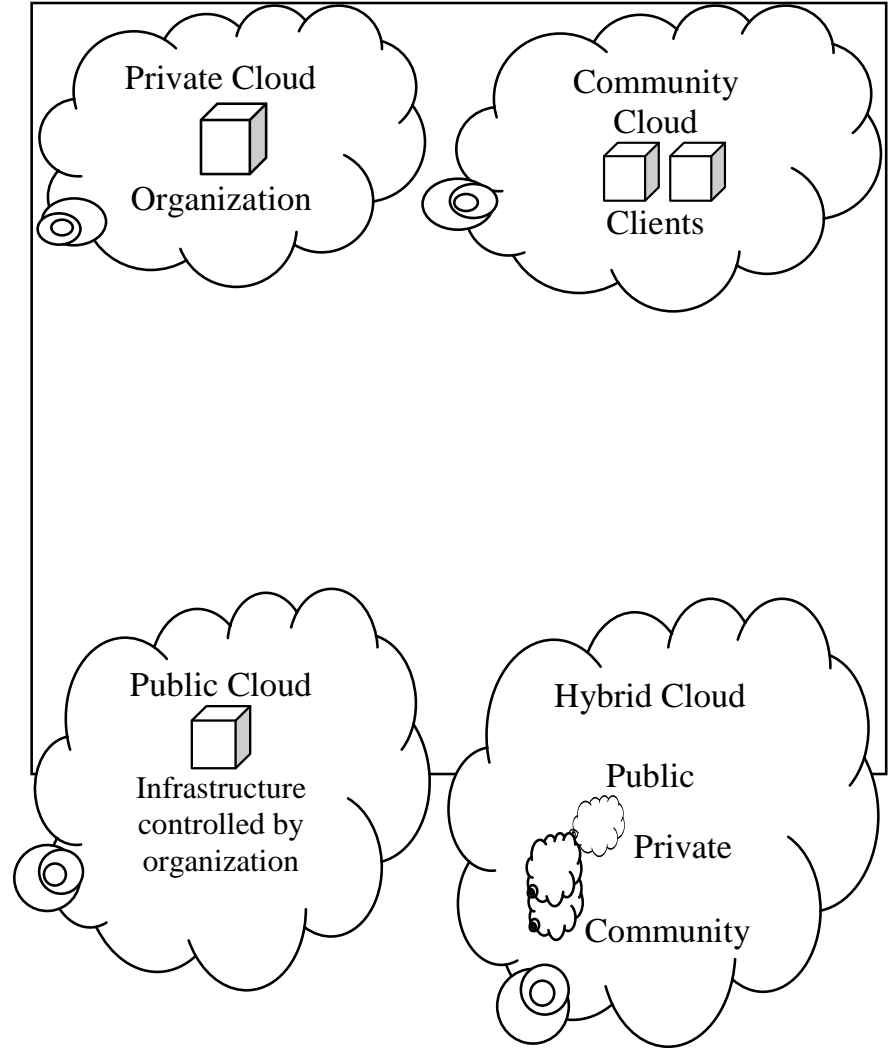

Figure 1: Deployment Models

\subsection{Public Cloud}

A cloud infrastructure is managed or controlled by an organization and they sell cloud services to public or companies. It is a model which allows user to access the cloud with the help of web browsers. This model is work on the principle of pay per use model. The public cloud computing [2] characterized one side by public availability of cloud service offering and on the other side by public network which provide connection with cloud service. The cloud service and cloud resources are obtained from very large resources pools that are shared by all end users. These IT factories which are built for running cloud computing systems, provides the resources. According to demand public cloud employ techniques for resource aggrandizement. These are pellucid for end users and indicate the conceivable foreshadow for the security of the system. Public cloud is less secure than other cloud computing models.

\subsection{Hybrid Cloud}

The combination of two or more than two clouds such as private, community or public known as hybrid cloud. It makes possible the transformation of data and application smoothly. Hybrid cloud uses a shared API to facilitate hybrid operations. In hybrid cloud organization can exploit cost benefits of public cloud and protect intimate data with the private cloud. All the features of deployment models are represented in table I.

\section{SERVICE MODEL}

\subsection{Software as a Service (SaaS)}

According to application service provider (ASP) model software as a service is a platform which supports multiple users at the same time by using resources of object code and database. A business user uses this type of services to complete their tasks. Email, office automation, website testing, services are provided by SaaS [3]. It is commonly accessed through a 
web portal and service oriented architecture based on web technology. Google apps, online email, IBM, Microsoft customer relationship management (CRM) etc are the basic examples of software as a service.

Table I: Summary of Deployment Models

\begin{tabular}{|l|l|l|l|}
\hline $\begin{array}{l}\text { Cloud } \\
\text { Models }\end{array}$ & Advantages & Disadvantages & Example \\
\hline $\begin{array}{l}\text { Private } \\
\text { cloud }\end{array}$ & $\begin{array}{l}\text { security, } \\
\text { control and } \\
\text { customization } \\
\text { robust data } \\
\text { segregation }\end{array}$ & $\begin{array}{l}\text { Cost, Capacity } \\
\text { management }\end{array}$ & $\begin{array}{l}\text { SOX, } \\
\text { HIPAA, } \\
\text { SAS 70 }\end{array}$ \\
\hline $\begin{array}{l}\text { Commun } \\
\text { ity cloud }\end{array}$ & $\begin{array}{l}\text { elasticity, } \\
\text { scalability }\end{array}$ & $\begin{array}{l}\text { security control, } \\
\text { cost, stored data } \\
\text { may be } \\
\text { accessible by } \\
\text { other } \\
\text { organizations }\end{array}$ & $\begin{array}{l}\text { google } \\
\text { managed } \\
\text { government } \\
\text { cloud }\end{array}$ \\
\hline $\begin{array}{l}\text { Public } \\
\text { cloud }\end{array}$ & $\begin{array}{l}\text { rapid } \\
\text { elasticity, } \\
\text { flexibility, } \\
\text { faster } \\
\text { deployment } \\
\text { time, } \\
\text { standardizatio } \\
\text { n to client }\end{array}$ & $\begin{array}{l}\text { Visibility and } \\
\text { efficiency, Not } \\
\text { suitable for } \\
\text { highly sensitive } \\
\text { data. }\end{array}$ & $\begin{array}{l}\text { Rackspace, } \\
\text { Amazon } \\
\text { Web } \\
\text { Services } \\
\text { (AWS), } \\
\text { Microsoft } \\
\text { Azure, } \\
\text { Google App } \\
\text { Engine }\end{array}$ \\
\hline $\begin{array}{l}\text { Hybrid } \\
\text { cloud }\end{array}$ & $\begin{array}{l}\text { security, } \\
\text { cost }\end{array}$ & $\begin{array}{l}\text { Complex } \\
\text { architecture, } \\
\text { technology } \\
\text { integration } \\
\text { challenges }\end{array}$ & Amazon s3 \\
\hline
\end{tabular}

\subsection{Platform as a Service (PaaS)}

In this service a new developed program is delivered to user via internet and servers. Developers create or deploy applications and services for users with the help of PaaS [10]. Service and application test, development, integration and deployment are some services which are provided by PaaS. The application of platform as a services are Microsoft Azure, Google application engine, IBM sMash etc.

\subsection{Hardware as a service (HaaS):}

Hardware as a service is same as licensing. In this service managed service provider (MSP) remotely monitors the hardware part of client and scales the expenditure or cost for clients. Microsoft Azure, Google application engine, IBM sMash are application of HaaS.

\subsection{Infrastructure as a Service (IaaS):}

It provides platform to users as computer environment or infrastructure as a service. Infrastructure as a service defines usage based payment scheme. In this user has to pay only for used service. This service provides fast delivery to user. This allows customer to pay as they grow. System manager uses LaaS [7] to create platforms for services and application test, development, integration. LaaS provides multiple services like virtual machines, operating systems, message queue, network, storage, CPU, memory, backup services. The IaaS applications are GoGrid and flexiscale. Figure 3 is representing all the services of cloud computing.

\section{CLOUD COMPUTING ISSUES}

Cloud computing rises lots of technical as well as legal issues [2] [7]. The presence of internet in the cloud computing rises same legal and technical issue of internet cloud computing is a big achievement of IT industry. Companies can aggrandize their business with very low cost but they want safe environment in cloud to maintain their downfall or prosperity.

A. SECURITY: In cloud computing cloud acts as a big black box. Nothing inside the cloud is visible to the client. Clients have no idea or control over what happens inside a cloud. multiple users and companies share their personal and private information and hackers can hack their data by APIs login in cloud which is big threat or risk for users .As security is one of the most difficult task to implement in cloud computing.

B. PRIVACY: Cloud computing uses virtual computing technology in which user's personal data saved in multiple virtual data centres instead of remain in same physical location. As users may leak their personal information when they access cloud computing services. All virtualized resources can be easily created or destroyed in any cluster.

C. AVAILABILITY: System can be able to accessed by authorized party in cloud computing infrastructure. Security branches, software and data can be accessed by authorized client. So cloud computing offers the open platform to user and attackers will become a big threat for clients.

D. CONFIDENTIALITY: Cloud computing provides easy way to access data and software to clients so cloud server are not honest for clients. There is a fear of loss of control over data for users.

E. RELIABILITY: Servers available in the cloud experiences some problems as resident servers. It is important to monitor services being provided using internal or third party.

F. PERFORMANCE BANDWIDTH COST: Cloud computing saves money of companies on hardware but they have to spend more for the bandwidth. Cloud provides low cost for smaller application but offers high cost for high data comprehensive application. Cloud requires sufficient bandwidth to deliver complex data over network due to which many companies waiting for a reduced cost before switching to the cloud.

\section{CLOUD AND GRID COMPUTING}

Cloud computing [6] uses multiple servers to provide virtual access of resources by internet and it is adopted object oriented programming concept to implement any system. Cloud computing used for small and large scale business activities. To perform complex calculations, high performance computer systems were used by inter connecting data links. As combined hardware and software infrastructure is known as grid computing. A grid [1] basically uses multiple processing computing units to perform a single task and single task is broken into multiple tasks and each machine assigned by a task. When sub tasks completed their task than they report to primary machine which controls all machine's task and they 
combined to produce output. The generation of technology is shown in figure 2 .

1. In grid computing multiple servers allocated to fulfill single task on the other hand in cloud computing virtualization of servers used in which one server is used to compute several task concurrently.

2. Grid computing is used for job execution. The execution of a program is performed for a limited period of time but cloud computing frequently supports long running services.

3. Cloud computing provides opportunity of unlimited scalability, cost saving, workload balancing but grid computing is a complex computing technology due to which cost increases. As cloud computing is more beneficial as compare to grid computing.

\section{CLOUD AND MOBILE CLOUD COMPUTING}

In public cloud, combination of hardware and software services provides to public users. It gives the flexibility to users to quickly add or scale up virtual servers and services according to their need. Cloud computing allows users to store the data like file, folder, documents in cloud area on internet and client can able to access all files and data wherever in the world but client should have a physical device to access data. Mobile computing provides physical device to clients like mobile phones, smart phones, tablets, laptops. These devices enable clients anywhere in the world because of the small size of the device. To take more advantages of cloud computing mobile users easily access multiple application and services over the wireless connection on web browser. As mobile cloud computing is the combination of mobile web application and cloud computing.

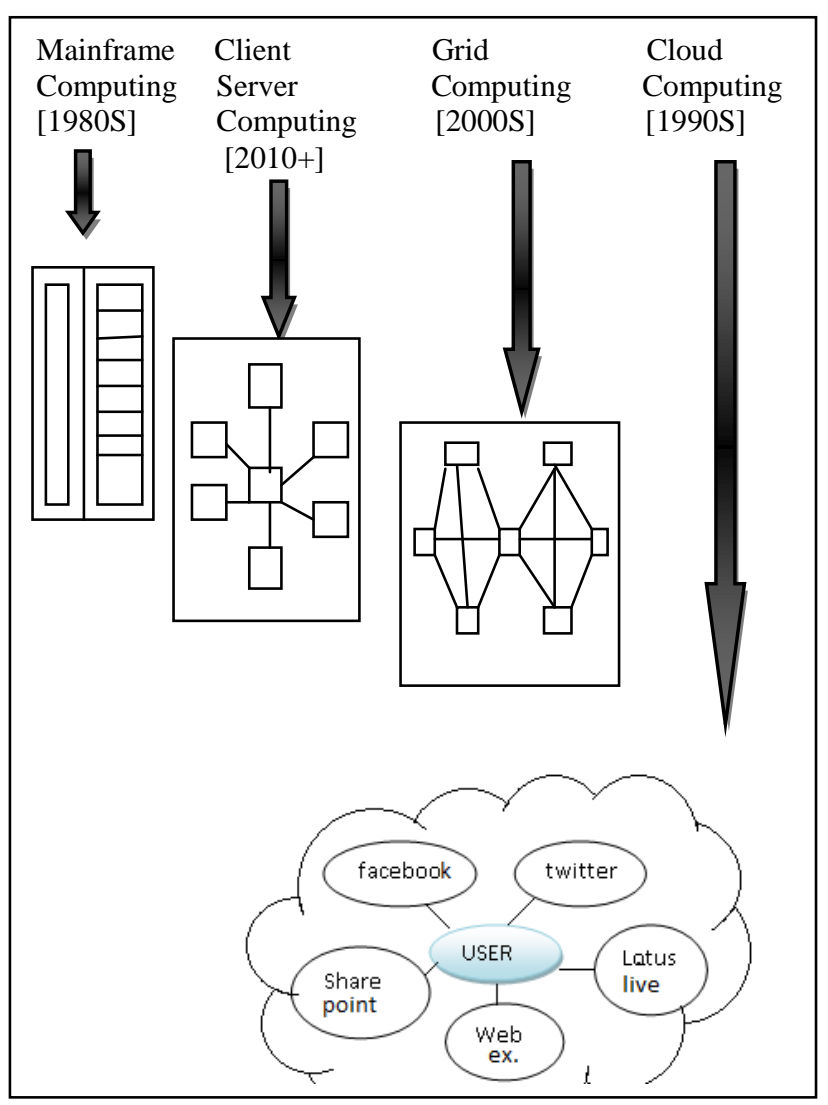

Figure 2: Technology Generation

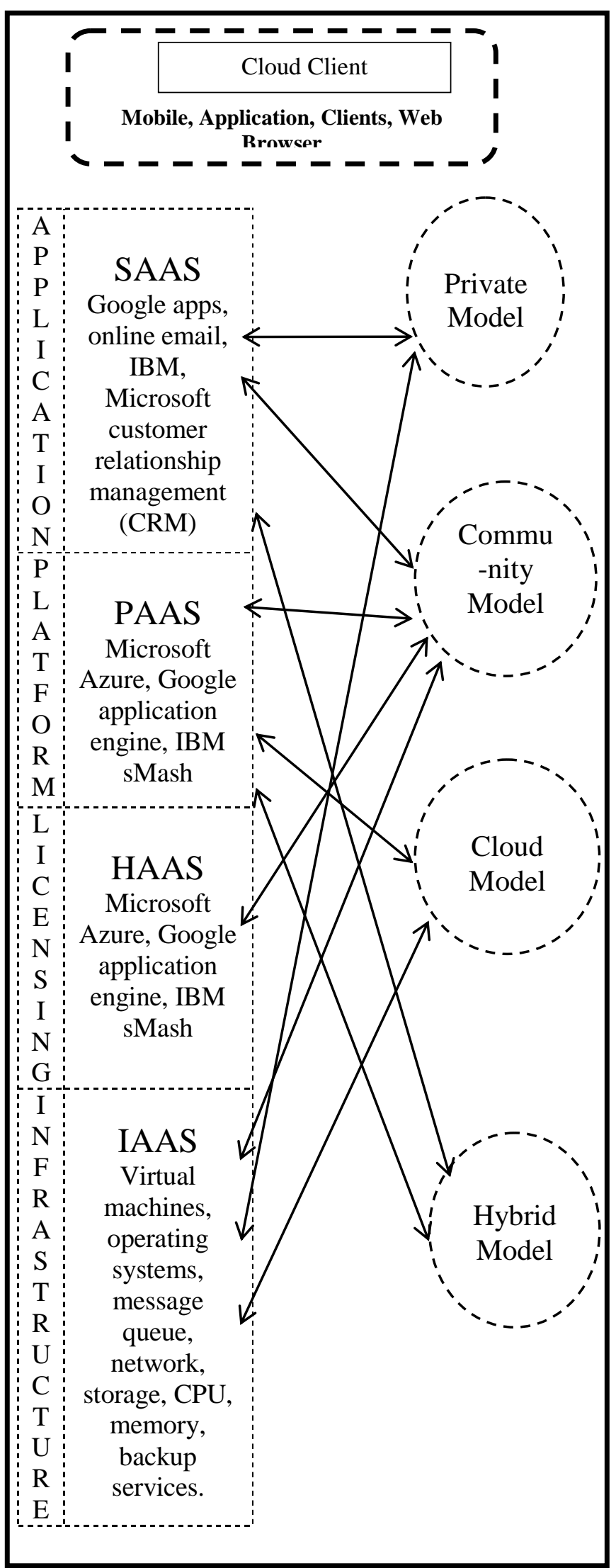

Figure 3: Cloud Services 
Mobile device do not need high level configuration system because multimedia services, transcoding and complex calculation processed on cloud. In mobile cloud computing [1] data is stored in the cloud which reduces the chance to loss data from mobile device as cloud provides remotely security service to mobile users. As mobile cloud computing is reliable to provide high data storage capacity, good processing power and security to mobile users. It provides lower bandwidth which is a technical issue in MCC. The bandwidth is determined by data distribution policy to share data between users. Which uses calling profile, signal strength profile, power profile to make a decision table of bandwidth by using markov decision process algorithm and that decision table decide whether or not a user have to download or receive data with other user due to bandwidth limitation.

In MCC offloading takes place which increases the battery life of the mobile device and application performance. But offloading consumes more energy as compare to local processing when the small size codes used. As offloading is a computing issue in MCC. In MCC security related issue are introduced in two categories - security for mobile users and security of data. As mobile computing has multiple advantages for mobile users and some technical issues also which are depend on both mobile communication and cloud computing.

\section{CONCLUSION}

Cloud computing provides a infrastructure with infinite computing capability, good scalability, service on demand also with some security, Privacy and legal issues. This paper indicates basic characteristics of cloud environment and important deployment models those are used to design cloud computing more effective. In this survey, discuss cloud services and issues of cloud computing as well as define some comparison points between cloud, mobile cloud and grid computing.

\section{REFERENCES}

[1] Niroshinie Fernando , Seng W. Loke , Wenny Rahayu, 2013, Mobile cloud computing: A survey, Future Generation Computer Systems, Vol. 29, pp: 84-106.
[2] Giuseppe Aceto, Alessio Botta, Walter de Donato, Antonio Pescap, 2013, Cloud monitoring: A survey, Computer Networks, Volume 57, Issue 9, PP: 2093-2115.

[3] Angela Lin, Nan-Chou Chen, April 2012, Cloud computing as an innovation: Percepetion, attitude, and adoption. , International Journal of Information management, Vol. 32, pp: 533-540.

[4] Md. Tanzim Khorshed, A.B.M. Shawkat Ali, Saleh A. Wasimi, 2012, A survey on gaps, threat remediation challenges and some thoughts for proactive attack detection in cloud computing. Future Generation Computer Systems, Vol. 28, pp: 833-851.

[5] Y. Ghanam, J. Ferreira and F. Maurer, 2012, Emerging issues and challenges in Cloud- A hybrid approach, Journal of software engineering and applications, vol. 5, no. 11, pp. 923-937.

[6] Guannan HU and Wenhao ZHU, 2011, A Dynamic Userintegrated Cloud Computing Architecture, International Conference on Innovative Computing and Cloud Computing (ICCC '11), pp: 36-40.

[7] Haoming Liang, Wenbo Chen and Kefu Shi, 2011, Cloud Computing: Programming Model and Information Exchange Mechanism, International Conference on Innovative Computing and Cloud Computing (ICCC '11), PP:10-12.

[8] Panagiotis Kalagiakos, Panagiotis Karampelas, 2011, "Cloud Computing Learning", IEEE Computer Society.

[9] T. Dillon, C. Wu and E. Chang, 2010, Cloud computing: Issues and challenges, 24th IEEE International Conference on Advanced Information Networking and Applications. pp. 27-33.

[10] Bhaskar Prasad Rimal, Eunmi Choi and Lan Lumb, 2009, A taxonomy and survey of Cloud Computing System, Fifth International Joint Conference on INC, IMS and IDC, pp: 44-51.

[11] M. A. Vouk, 2008, Cloud computing Issues, research and implementations, Journal of computing and information technology, Vol. 16, no. 4, pp. 235-246. 